
FciDioc

\title{
Application of The Bi-Level Stairs Climbing as Alternative of Respiratory Rehabilitation Training in Dyspneic Smokers
}

\author{
Research Article
}

Gimenez $\mathrm{M}^{1 *}$, Saavedra $\mathrm{P}^{2}$, Lantarón $\mathrm{EM}^{3}$, Sierra $\mathrm{V}^{1}$, Polu $\mathrm{E}^{4}$, Servera $\mathrm{E}^{5}$

${ }^{1}$ Departments of Physical Medicine and Rehabilitation and Respiratory Physiotherapy, University Hospital of Gran Canaria Dr. Negrín, Bco de la Ballena s/n, University of E-35020- Las Palmas de Gran Canaria, Spain.

${ }^{2}$ Department of Mathematics and Informatics, University Campus of Tafira, University of Las Palmas de Gran Canaria, Spain.

${ }^{3}$ Faculty of Physiotherapy - Campus of Pontevedra, A Xunqueira s/n. 36005- Pontevedra. University of Vigo, Spain.

${ }^{4}$ Institute of Rehabilitation, University of Nancy, Nancy, France.

${ }^{5}$ Faculty of Medicine of Valencia, and Clinical Hospital University of Valencia INCLIVA, Spain.

\section{Abstract}

Background: Physiological studies using standardized bi-level endurance stairs climbing in respiratory rehabilitation are rare. The authors have developed and proved that the "Bi-level Stairs climbing" (BiSC [13]) compared to $\mathrm{VO}_{2} \max$ could be an optimal rehabilitation training program.

Goal of this work: Help define which submaximal level of BiSC is the closest in terms of physical effort to the 30 minutes training sessions.

Methods: BiSC has indeed numerous advantages: it can be practiced almost anywhere, well accepted and tolerated by healthy trained or not and smokers. Furthermore, stairs with one flight only could be used effectively if the procedure is adequately followed. This paper describes a precise protocol to answer this question.

For this purpose, a target sample size of 25 unmedicated, voluntaries active male smokers from 18 to 66 years and normal body mass index (BMI) was pre-determined to achieve $80 \%$ power.

Results: The number of cycles indicates repeating the sub-maximal series climbing and down as the represented number, for instance the series BiSC-5 indicates climbing and down five consecutive floors, and so on for the progressive floor until the maximal BiSC-10. The protocol and the timings of either the exercise or the measures are fully detailed in the design and method section.

The results and conclusion of this work concern the determination of a « best, safe, and optimal » training program, using stair climbing technique, for a group of ordinary smokers. All subjects could perform the BiSC- 5 training for 30 minutes.

Conclusion: After comparing different cycles of stair climbing, the authors agree and propose the BiSC-5 (with 5 flights) program as the most useful, economic, effective and aerobic training exercise, at least for the smokers with normal spirometry. The five parameters of the tailored BiSC-5 correspond well with those of VAT on cycle ergometry making the BISC-5 a good alternative for effective exercise training both in the hospital and at home.

Keywords: Bi-Level Stairs Climbing; Dyspnea; Spirometry; Maximal Ventilation; Sub Maximal and Maximal Exercises, $\mathrm{VO}_{2}$, $\mathrm{V}_{\mathrm{E}}$; VAT: Peak Work Rate; $\mathrm{V}_{\mathrm{T}}$; HR; RR; Arterial Blood Pressure; $\mathrm{SpO}_{2}$.

\section{Introduction}

Structured exercise rehabilitation programs of six to eight weeks duration have been reported to improve quality of life, increase exercise tolerance, and boost maximal oxygen consumption $\left(\mathrm{VO}_{2} \max \right)$ by 20 to $40 \%$.and endurance by $60 \%$ [3-5].
Significant benefits can be gained and maintained with just 45 minutes of daily maximal training on cycloergometry [3-5] for subjects with pulmonary problems, in respiratory rehabilitation centers. Unfortunately most people who cannot afford it at home, stop exercising after they leave medical care and assistance; thus reducing the achieved benefits $[4,5]$.

\footnotetext{
*Corresponding Author:

Dr. Manuel Gimenez, MD, PhD,

Departments of Physical Medicine and Rehabilitation and Respiratory Physiotherapy, University Hospital of Gran Canaria Dr. Negrín, Bco de la Ballena s/n, University of E-35020- Las Palmas de Gran Canaria, Spain.

E-mail: Gimen3@hotmail.com

Received: March 06, 2017

Accepted: June 19, 2017

Published: June 22, 2017

Citation: Gimenez M, Saavedra P, Lantarón EM, Sierra V, Polu E, et al., (2017) Application of The Bi-Level Stairs Climbing as Alternative of Respiratory Rehabilitation Training in Dyspneic Smokers. Int J Resp Dis Care Med. 2(3), 22-27. doi: http://dx.doi.org/10.19070/2577-4409-170005
}

Copyright: Gimenez $\mathbf{M}^{\circ}$ 2017. This is an open-access article distributed under the terms of the Creative Commons Attribution License, which permits unrestricted use, distribution and reproduction in any medium, provided the original author and source are credited. 
If motivation is left apart, equipments pricing and/or shortness of time can be easily faced with low-cost and low-tech alternatives such as just efficient walking, cycling, stairs climbing and/or using two-step stool [7]. Besides, many European chest physicians and oncologists often use stairs climbing to screen patients for impaired pulmonary function and subrogate of $\mathrm{VO}_{2} \max$ prior to surgery $[8,9]$.

Our team has developed a particular type of stairs climbing called "Bi-level Stairs climbing" (BiSC) [13] which will be used in this study. Its usefulness comes from its simplicity as it needs only one flight; it combines an ascent and descent phase timed; and during experimentation and teaching the patient, in the stairs of the rehabilitation center, it is easy for medical professionals to monitor and supervise the process and the patient as they watch him all along.

Existing protocols of stairs vary greatly though, and the lack of target reference values has limited its more widespread adoption discussed recently [6]. At present time, unfortunately no physiologic study on standardized bi-level endurance stairs has been yet published although some authors made some propositions for energy assessment during training sessions more for cicloergometry than for stairs $[5,7]$.

In order to bring a contribution to the questioning above, this paper reports: (a) The assessment of five clinical/functional values; (b) The analyze of the differences in percentage between "sub maximal" levels [13] of the BiSC "exercise endurance" in relation to the maximum level BiSC possible, in order to define the most favorable and sustainable protocol for training with BiSC [7]; (c) A procedure to help define which level of BiSC is the closest in terms of physical effort to the 30 minutes training sessions, (d) Comparing functional respiratory parameters with those of ventilatory anaerobic threshold (VAT) and peak work rate (PWR) on cycle ergometry, i.e. this can be called "the optimal stair climbing training program using BiSC”.

\section{Methods}

This study was approved by the institution's ethics committee and was conducted according to Declaration of Helsinki standards. All studied subjects gave informed consent.

Unmedicated, voluntaries active male smokers were recruited [10]. A target sample size of 25 subjects was pre-determined to achieve $80 \%$ power. Inclusion criteria were: age 18 and older after 66 years, having normal body mass index (BMI), absence of bronchospasm based on European Community for Steel and Coal questionnaire and observation at rest and during exercise to $\mathrm{VO}_{2} \mathrm{max}$ on cycle ergometry [12]. Exclusion criteria were insufficient cardiopulmonary, neuromuscular, medical, or physical capacity to permit strenuous exercise, abnormal spirometry [12], chest radiographs, or electrocardiogram $[5,10,11]$ evidence of bronchospasm, or not signing consent.

The maximum of three values was recorded only for slow vital capacity (VC), forced expiratory volume in the first second (FEV1), and maximum voluntary ventilation (MVV) were taken. Minute ventilation (VE), tidal volumes (VT), respiratory rate (RR) and respiratory exchange, that is, oxygen uptake $\left(\mathrm{VO}_{2}\right)$ and carbon di- oxide output $\left(\mathrm{VCO}_{2}\right)[5,7]$ were measured at the VAT and during the maximal exercise cycle ergometry protocol PWR, 30W/3min, by Jaeger's Oxycon Champion (Jaeger GmdH \& Co., Wurzburg, Germany), calibrated before and after each use [5, 11]. A bronchodilator (i.e. Salbutamol $0.2 \mathrm{mg}$ ) was administered for a second round of measurements to establish any effect of bronchodilatation.

Maximal cycle ergometry (1000S, Medifit Inc, Maarn, The Netherlands) was undertaken for all subjects at increments of 30 watts $/ 3$ minute with the subjects on a cardiac monitor (Multiscriptor EK; Hellige-France Inc, Strasbourg, France) [5, 11]. The PWR was that achieved during the last full 3 min before exhaustion and the VAT was determined $[5,11]$. The $\mathrm{VO}_{2} \max$ was recorded as the $\mathrm{VO}_{2}$ over the last $30 \mathrm{sec}$ before exhaustion [5, 11]. Oxygen pulse $\left(\mathrm{O}_{2} \mathrm{P}=\mathrm{VO}_{2} / \mathrm{HR}\right)$ was calculated at rest and at each ergometry increment. Exertional Dyspnea (ED), leg pain (LP) [12], pulse Oxymetry saturation $\left(\mathrm{SpO}_{2}\right)$ and heart rate $(\mathrm{HR})$ were monitored (Biox Pulse Oximeter; Ohmeda, Louisville, CO).

Exertional dyspnea and leg pain were measured by modified Borg scale [12], a 0-10 Liker scale with 10 representing most severe [12]. They were measured after each flight increment of BiSC, and after the cycle exercise to VAT and to PWR [5].

Bi-level Stairs climbing (BiSC) [13], after the $\mathrm{VO}_{2}$ max was determined by cycle ergometry, 1 to 3 days later the 25 consenting subjects performed the BiSC over maximal Floors (BiSC-10) [13]. A 1-story building was used with uniform 3 meter flights with handrails. There were 20 steps per flight with steps of $15 \mathrm{~cm}$ in height and $25 \mathrm{~cm}$ in breadth. The subjects were instructed to climb until exhaustion for the BiSC max. The BiSC stair climbing rate was standardized to $12 \mathrm{sec}$ per flight $[11,13]$ paced by metronome $[7,13]$. For BiSC, the flights were also descended in $8 \mathrm{sec}$ paced by metronome and followed by $10 \mathrm{sec}$ of rest for clinical assessment, for $30 \mathrm{sec}$ total per flight. The total numbers of consecutive flights settle for each protocol was record [13]. Arterial Blood pressures were measured at the end of all maximal tests. A specialized physiatrist and a physiotherapist collected the data during the rest periods [13].

How to use the Bi-level Stairs climbing as a respiratory rehabilitation alternative?. Since the protocol with the best outcomes observed recently was the BiSC-10 [13] and every subject could negotiate nine sub maximal flights [13], it was studied for its aerobic training potential over a sub maximal flight for maintenance exercise training. In the sub maximal flight BiSC-5 protocol (Figure 1), the subjects climbed and descended a single flight in $20 \mathrm{sec}$ plus $10 \mathrm{~s}$ for measurements of the five variables with a total of 30 s per flight, as the sub maximal level and maximal times (i.e. one cycle), climbing and down one floor, with 1 minute rest periods between the five flights repetitions of each cycles $[11,13]$. The number of cycles indicates repeating the sub-maximal series climbing and down as the represented number, for instance the series 5 indicates climbing and down five consecutive floors, and so on for the progressive floor until the last supported series, wherever the subjects repeated the numbers of Flights [13]. (See later on the results).

After obtaining the mean values of the five variables in the ten series, the second step will be to know which of those five variables, isolated or associated, can guide to choose the most suitable and best supported level of training for the subjects concerned. 
To do this, the values of ratios of each submaximal series related to maximum, and expressed in percents, will be calculated. Those ratios are then compared with their respective correspondent values of VAT and PWR obtained on cycle ergometry [11, 20-22]. in order to determine which parameter is best adapted.

\section{Statistical Analysis}

The measured responses were summarized as means and standard deviations or medians and interquartile ranges (IQR) based on the data distribution [14]. T-test and Wilcoxon test for independent variables were used to compare BiSC-10 and BiSC-5 data [14]. Statistical significance was set at $\mathrm{p}<0.05$. The arterial blood pressure and heart rate values at rest, after the $10^{\text {th }}$ floor of the BiSC10 , and both at BiSC- $5^{\text {th }}$ floors: 1) as a test, or 2) as 30 min session training; and also at PWR of the incremental cycle ergometry, were compared. The means were also estimated by means of confidence intervals at $95 \%$. All statistical analyses were performed using the R package, version 3.0.1 (R Development Core Team, 2013) [15].

\section{Results}

At rest, at the baseline, the mean values for HR are: $84 \pm 4.35$ pulsations per minute; for peripheral saturation, $\mathrm{SpO}_{2}=95 \% \pm 0.74$, and for $\mathrm{RR}=23 \pm 3.4$ breaths $/ \mathrm{min}$. All subjects could perform the selected BiSC-5 during 30 minutes session training.

Table 1 shows the statistical description of the sample: i.e. means and standard deviations of their physical characteristics and age; the BMI values indicate that there are no obese in the group. The spirographic values are in the normal range; however that of $\mathrm{VO}_{2}$ max and of ventilatory anaerobic threshold (VAT) are reduced.

Table 2: Illustrates the mean percentage values of the four variables as a Function of Flights Climbed in percentage of the ratios observed. It also shows the comparisons between the four variables of the BiSC- 10 with two submaximal BiSC- 5 as a test and as a 30 min session training. It could be seen that exertional dyspnea and leg pain were significantly lower $(\mathrm{p}<0.05)$, during the submaximal BiSC- 5 over 5 flights, than for the BiSC- 10 flights, as well as the comparison of the heart rate and breathing rate. The two sub maximal BiSC-5 have significantly lower HR and RR than those of maximal BiSC-10 ( $\mathrm{p}<0.05)$, However, the ratio HR at the BiSC- 5 training over HR of the BiSC-10 is significantly higher $(69.7 \%, \mathrm{p}<0.05)$.

Figure 1 shows a Schematic representation of the BiSC- 5 climbing stairs protocol, with the corresponding climbing, down and total times (30s) for every one of the climbing Flight, plus $1 \mathrm{~min}$ rest in between the nine cycles.

Table 3 presents the comparisons between the arterial blood pressures and heart rate during exercises at different levels of exertion. It was observed that: (1) BiSC-10 over PWR, the three variables are significantly lowers for the BiSC-10 ( $p<0.05)$; (2) The BiSC-5 training at its $9^{\text {th }}$ cycle over PWR, only the systolic AP and the HR are lowers on the 9 cycle $(\mathrm{p}<0.05)$.; (3) On BiSC-5 training, the cycle 1 over the cycle 9 , the three variables are significantly lowers at the first cycle $(\mathrm{p}<0.05)$. The highest values of arterial pressure are on the PWR.

Table 1. Anthropometric, Pulmonary and Ergometry Variables.

\begin{tabular}{|c|c|}
\hline Variables at Rest and Exercise & Active Smokers N $=25$ \\
\hline Age, years & $46.9 \pm 2.9$ \\
\hline Weight, kg & $75.5 \pm 7.4$ \\
\hline Height, $\mathrm{cm}$ & $175.2 \pm 5.0$ \\
\hline BMI, $\mathrm{Kg} / \mathrm{m}^{2}$ & $24.6 \pm 1.7$ \\
\hline VC L & $5.27 \pm 0.49$ \\
\hline $\mathrm{VC} \%$ & $121 \pm 11$ \\
\hline FEV1 L & $3.85 \pm 0.40$ \\
\hline FEV1 \% & $127 \pm 13$ \\
\hline FEV1/VC \% & $73.1 \pm 2.7$ \\
\hline $\mathrm{MVV} \mathrm{L/min}$ & $154 \pm 16$ \\
\hline $\mathrm{VO}_{2} \max ($ cycle) $\mathrm{mL} / \mathrm{min})$ & $2508 \pm 327$ \\
\hline $\mathrm{VO}_{2} \max (\mathrm{mL} / \mathrm{kg} / \mathrm{min})$ & $33.2 \pm 3.5$ \\
\hline PWR (Watts) & $180 \pm 21.9$ \\
\hline PWR (Heart Rate) & $166 \pm 4.3$ \\
\hline $\operatorname{VAT}\left(\mathrm{VO}_{2}\right)$ & $1283 \pm 271$ \\
\hline$\left(\mathrm{VO}_{2} \mathrm{VAT} / \mathrm{VO}_{2} \max \%\right)$ & $51.2 \pm 4.3$ \\
\hline VAT (HR beats/min) & $119 \pm 5.9$ \\
\hline (HR VAT/HR max \%) & $72 \pm 4$ \\
\hline VAT (RR) (Breathes/min) & $27.08 \pm 3.85$ \\
\hline VAT (ED) (Borg scale) & $3.5 \pm 1.21$ \\
\hline VAT (Leg Pain) (Borg scale) & $3.88 \pm 0.89$ \\
\hline
\end{tabular}

Values are presented as means $\pm \mathrm{SD} ; \mathrm{BMI}=$ body mass index; $\mathrm{VC}=$ slow vital capacity; FEV1 $=$ forced expiratory volume in the first second; $\mathrm{MVV}=$ maximal voluntary ventilation; $\mathrm{VO}_{2} \max =$ maximal Oxygen consumption; PWR = Peak Work Rate on cycle ergometry; VAT = Ventilatory anaerobic threshold; HR = Heart rate, beats $/ \mathrm{min} ; \mathrm{RR}=$ Respiratory rate, breathes $/ \mathrm{min} ; \mathrm{ED}=$ Exertional Dyspnea, Borg scale (0-10); LP = Leg pain, Borg scale $(0-10)$ 
Table 2. Four variables as a Function of Flights Climbed. Mean percentage of the ratios: number of Floor/maximum Floor observed.

\begin{tabular}{|c|c|c|c|c|}
\hline BiSC series Floor & HR HR & RR RR & ED ED & LP LP \\
\hline Floor 3 & $58.4 \%$ & $44 \%$ & $19.7 \%$ & $23 \%$ \\
\hline Floor 4 & $64.2 \%$ & $49.4 \%$ & $29.85 \%$ & $31.5 \%$ \\
\hline Floor 5 & $69.7 \%$ & $55.3 \%$ & $39.5 \%$ & $36.5 \%$ \\
\hline Floor 6 & $74.8 \%$ & $62.7 \%$ & $53.9 \%$ & $55.8 \%$ \\
\hline Floor 7 & $80 \%$ & $70.5 \%$ & $66 \%$ & $71 \%$ \\
\hline Floor 8 & $84.5 \%$ & $79.8 \%$ & $80 \%$ & $82 \%$ \\
\hline Floor 9 & $92 \%$ & $89.5 \%$ & $90.8 \%$ & $92.2 \%$ \\
\hline
\end{tabular}

Bi-level stairs climbing; HR = Heart rate, beats $/ \mathrm{min} ; \mathrm{RR}=$ Respiratory rate, breathes $/ \mathrm{min} ; \mathrm{ED}=$ Exertional Dyspnea, Borg scale $(0$ 10); LP = Leg pain, Borg scale $(0-10) ;{ }^{*} \mathrm{p}<0.05 ;{ }^{* *} \mathrm{p}<0.01 ;{ }^{* * *} \mathrm{p}<0.001$

Table 3. Comparison of Blood Pressures and Heart Rates at Rest and During Exercises of different levels.

\begin{tabular}{|c|c|c|c|}
\hline Rest and Exercises (n = 25) & $\begin{array}{c}\text { Systolic BP } \\
\mathbf{m m H g}\end{array}$ & $\begin{array}{c}\text { Diastolic BP } \\
\mathbf{m m H g}\end{array}$ & $\begin{array}{c}\text { Heart rate } \\
\text { Beats/min }\end{array}$ \\
\hline At rest & $142.2 \pm 4.13$ & $80.5 \pm 2.6$ & $80.5 \pm 4.31$ \\
\hline E1) BiSC-10 Series 10 Flights & $166 \pm 3.1$ & $76.3 \pm 2.2$ & $166.7 \pm 5.81$ \\
\hline E2) BiSC-5 training cycle 9 & $164 \pm 3.86$ & $80 \pm 2.62$ & $152.8 \pm 5.43$ \\
\hline E3) BiSC-5 training cycle 1 & $153 \pm 4.85$ & $76 \pm 2.50$ & $115 \pm 11.9$ \\
\hline E4) cycle-PWR (cycle ergometry) & $184.6 \pm 24.5$ & $79.58 \pm 2.44$ & $167.7 \pm 5.81$ \\
\hline
\end{tabular}

$\mathrm{E}=$ Exercise $\mathrm{BP}=$ Blood Pressure; BiSC-10 = Bi-level stair-climbing 10 floors; BiSC-5 over 5 Flights = Bi-level intensity stairclimbing over 5 flights; PWR = Peak Work Rate. Data are means \pm SD. Significant difference $(\mathrm{p}<0.05)$. The BiSC-5 training cycle 1, corresponds to the series 1 of the BiSC-5, and to the series 9 of the BiSC-5.

Figure 1. Bi-level Stairs Climbing as a 30 min Training Session in Smokers Men.

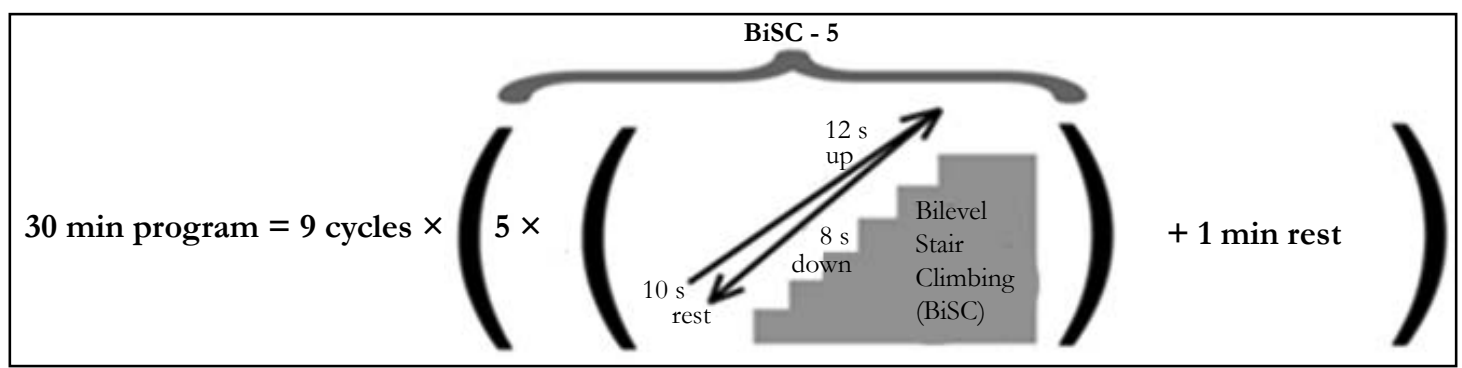

Schematic representation of the BiSC-5 climbing stairs protocol, with the corresponding climbing, down and total times (30s) for every one of the climbing Flight, plus 1 min rest in between each cycles.

Table 4. Comparison of $\mathrm{SpO}_{2}$ on three BiSC tests with VAT and PWR at Cycle Ergometry.

\begin{tabular}{|c|c|c|c|c|c|c|}
\hline $\boldsymbol{S p}_{\boldsymbol{p}} \boldsymbol{\boldsymbol { O } _ { 2 }}$ & Rest & VAT Test & BiSC-5 Test & BiSC-5 Training & BiSC-10 Test & PWR Watts \\
\hline $\mathrm{Mean}$ & 95 & 96.5 & 96.29 & 95.4 & 95.3 & 94.7 \\
\hline $\mathrm{SD}$ & 0.74 & 1.08 & 1.06 & 0.67 & 0.81 & 1.1 \\
\hline
\end{tabular}

$\mathrm{SpO}_{2}=$ Peripheral Oxygen Saturation; VAT $=$ Ventilatory Anaerobic Threshold; BiSC = Bi-Level Stairs Climbing: PWR = Peak Work Rate.

Table 4 shows the comparisons of the mean and standard deviation of the peripheral $\mathrm{O}_{2}$ saturation $\left(\mathrm{SpO}_{2}\right)$ during the three tests of of BiSC: BiSC-5 as test; and BiSC-5 as 30 min session training and maximal BiSC-10; also during the Ventilatory anaerobic threshold and PWR measured by cycle ergometry (30W/3 min).

\section{Discussion}

The objectives of this study were to assess the possibility to achieve a training session of 30 minutes with a sub maximal $\mathrm{Bi}-$ level Stairs climbing [13]. The results observed confirm that the behavior of the five variables suggest that the following three objectives have been validated: 
[a.] The subjects were able to achieve the ten sets of stairs (Table 2).

[b.] Based on these data, it could be objectively establish the selection of the level of targeted training (Table 2).

[c.] All subjects were able to achieve the nine cycles of $30 \mathrm{~min}-$ utes session with the BiSC-5, almost aerobically (Tables 2 and 3).

For obvious reasons, the set levels 1-4 are excluded for the session training because they are in fact rather submaximal aerobic level, and easy to accomplish. On the contrary, in the sets of 7-10 most variables are above between 70 and $80 \%$ of the maximum level which excludes them for their anaerobic state during the 30 minute session $[10,11]$. Consequently, only five and six cycles (Table 2) will be analyzed, to know which of them have a better approach close to the ventilatory anaerobic threshold (VAT), that is the ratios over the tenth cycle with the more physiologic submaximal aerobic and favorable performance.

From Table 2 it appears, as a result, that the set 5 is the best that suits this analysis: three of these variables are below $55 \%$. Ratio analysis shows that, for the five series, respiratory rate is lower and, both subjective variables (ED and LP) are below 40\%. Finally, the $\mathrm{SpO}_{2}$ is in the normal range, susgesting clear aerobiosis. However, only the HR seems moderately high, but still showing that there is $30 \%$ of the heart rate reserve (Tables 2 and 3). Even so, comparing the average values of the 5 Series for the HR (Table 2), with that of ventilatory anaerobic Threshold (Table 1), the difference is not significative. In addition, the mean values of ED is $19 \%$ lower and the LP is only $10 \%$ higher than these of VAT. Doubt on the energétique target level between sets 5 and 6 , has guided our choice on the Series 5 (Tables 2 and 3). Thus, level-5 story was the one that most corresponded with percentages measured in ventilatory anaerobic threshold, so it could be used for demostration (Tables 2 and 3). Table 2 illustrates that there are a discrete difference, for the four variables $(\mathrm{p}<0.05)$ in between both submaximal BiSC-5 (test and training). In addition the Leg Pain is, frequently, significantly higher than the Exertional Dyspnea $(p<0.01)$. The differences are higher between the BiSC-10 with both submaximal levels (BiSC- 5 test and BiSC- 5 session training, $\mathrm{p}<0.001)$. So we followed the description seen in Figure 1 for the training session with the series BiSC-5.

Moreover, on the BiSC-5 training sessions, no subjects' exercise parameters exceeded acceptable targets; 5 flights of BiSC- 5 was well tolerated by all subjects; and the energy output achieved corresponded well with previously and actually described cycling exercise, that achieved ventilatory anaerobic threshold protocol (VAT, Table 1) [5, 13]. Thus, the BiSC-5 session training is safe and easy to understand, and is an aerobic session of training.

Also, achieving $69 \%$ of the heart rate, observed at the $\mathrm{VO}_{2} \mathrm{max}$, demonstrates that maximal stair climbing training during $30 \mathrm{~min}$ utes can be individual high intensity exercise (Tables 2 and 3). Besides, the arterial pressure, systolic and diastolic, were also roughly in the order of these values, thus, it was better tolerated and achieved aerobic training levels at higher respiratory rates $(\mathrm{p}<0.05)$.

Stair climbing can provide aerobic training without specialized equipment [16-19] and has been recommended to alleviate
America's and Europe's obesity epidemic [1, 6, 9, 17]. However, studies to date have been limited by heterogeneity of stair (as "material for measurement on terms of intensity and altitude") and as climbing protocols, because of inadequate control of confounding factors $[9,17-19]$. This study overcame some of these limitations. First, the BiSC is original [13], and is proposed as test and as a session of stairs training; Second, this was a homogenous group of 25 highly motivated unmedicated active male smokers, of similar age and health status, with similarly low maximal exercise capacity because of the smoking $[5,11]$. Third, there were strict inclusion and exclusion criteria. Four, The subjects were methodically instructed and the exercise strictly regimented $[5,11]$. Five, individual $\mathrm{VO}_{2}$ max and Ventilatory anaerobic Threshold parameters were verified by cycle ergometry. Six, a fixed stair climbing rate was implemented $[7,11]$. Seven, in addition, the protocols were repeated to ensure good reproducibility $[11,13]$.

Bi-level cycling exercises have been reported to achieve greater $\mathrm{VE}$ and $\mathrm{VO}_{2}$ in healthy subjects [11, 20-23], chronic obstructive pulmonary disease (COPD) [11, 22, 26], and in pulmonary [26] and cardiac [23] transplant patients [23-26], than has fixed intensity exercise [23-26]. Our previous comparisons of maximal BiSC10 over ten flights max with the continuous FiSC- 8 over 8 flights max [13] were consistent with this but BiSC-10, and especially BiSC-5 over 5 flights, had a particularly desirable cardiovascular profile $[7,10,11]$ and was best tolerated [10].

Similar to Bi-level SWEET [20-23], BiSC-5 over 5 floors training involves brief intervals of positive work (i.e., 12 seconds of ascend), negative work (i.e., 8 seconds of descend) and produces a high respiratory rate (Table 2 ). The energy expenditure for BiSC-5 over 5 floors for 30 minutes $(82.5 \mathrm{~kJ}$ or $1.10 \mathrm{~kJ} / \mathrm{kg}$ is only $11.6 \%$ that of SWEET for 45 minute $(713 \mathrm{~kJ}$ or $10.18 \mathrm{~kJ} / \mathrm{kg}$, i.e. by cycle-ergometry) $[11,22]$ so individuals should be able to easily maintain their conditioning using either BiSC- 5 over 5 floors or the SWEET [5, 22, 23]. Even maximal BiSC-10 over 10 flights was done at lower heart rate than reported values of exercise to $\mathrm{VO}_{2}$ max or PWR (Table 3) $[5,11,22,26]$.

Six minute walks, too, is often used to estimate aerobic exercise capacity $[27,28]$. Continuous walking has also been recommended by some health professionals for endurance training [6]. However, 6 minute walk studies have up to $42 \%$ variability $[7,27,28]$ and have not been standardized for endurance training because the speed was not evaluate.

Many studies on stair climbing have been analyzed and they all suffer from lack of standardizing way to use the stairs [29]. Some attempts have been made such as the most frequently used Stair Climbing Test (SCT) developed for the first time in 1955. Many authors have developed their own variation of it, (like BISC used for dyspneic smokers [13]) [29].

The physical condition of each subject enrolled is first checked for safety and acceptability to perform the stair exercises (in terms of intensity and time). Indeed the first phase of the process, is to obtain their maximum peak work rates. The maximum peak work rates are measured at the hospital through cycle ergometry test. Subject with severe manifestations of coronary heart disease are excluded from the study.

The results and conclusion of this work concern the determina- 
tion of a « best, safe, and optimal » training program, using stair climbing technique, for a group of ordinary smokers. Finally, this study have esteemed the indications of measuring treatment fidelity in respiratory rehabilitation by Hildebrand et al., [31].

\section{Conclusion}

BiSC- 5 program over 5 floors may be useful to provide economical, effective aerobic exercise for smokers with Dyspnea and normal spirometry.

\section{Acknowledgments}

We thank Lahoucine Benhamgar, PhD, epidemiologist researcher, for scientific and statistical advising, assistance and constructive criticism throughout the study; Jean Lonsdorfer MD, PhD, Maria Angeles Montoliu, MD, PhD, Belen Alonso, MD, PhD for clinical help and advice; Encarnación Abril, Mari Carmen Lareo and Andrés Aranda PT, for their participation in the clinical trial and implementation of physical protocol techniques; Juan Verona and Maeva Sossi for help with graphics and drawings; and the 25 smokers who faithfully followed the complete protocol.

\section{References}

[1]. Sword, David O (2012) Exercise as a Management Strategy for the Overweight and Obese and Obese: where does resistance exercise Fit in ? Strength \& Conditioning J. 34(5): 47-55.

[2]. National Center for Chronic Disease, Prevention and Health Promotion and Centers for Disease Control and Prevention (1996). Physical Activity and Health: A Report of the Surgeon General. June, 2009.

[3]. Varga J, Porszasz J, Boda K, Casaburi R, Somfay A (2007) Supervised high intensity continuous and interval training vs. self-paced training in COPD. Respir Med. 101(11): 2297-2304.

[4]. Casaburi R, Patessio A, Ioli F, Zanaboni S, Donner CF, et al., (1991) Reduction in exercise lactic acidosis and ventilation as a result of exercise training in patients with obstructive lung disease. Am Rev Respir Dis. 143(1): 9-18.

[5]. Gimenez M, Saavedra P, Martin N, Polu JM, López D, et al., (2012) Bilevel exercise training and directed breathing relieves exertional dyspnea for male smokers. Am J Phys Med Rehabil. 91(10): 836-845.

[6]. World Health Organization (2010) Recommandations mondiales sur l'activite physique pour la sante. Geneva. 58.

[7]. Gimenez M, Saavedra P, Martin N, Lantarón EM, Polu E, et al., (2014) Two Step Stool Aerobic Training for Smokers. Am J Phys Med Rehab. 93(7): 1-9.

[8]. Charloux A, Brunelli A, Bolliger C, Rocco G, Sculier JP, et al., (2009) Lung function evaluation before surgery in lung cancer patients: how are recent advances put into practice? A survey among members of the European society of thoracic surgeons(Ests) and of the thoracic oncology section of the European respiratory society(ERS). Interact Cardiovasc Thorac Surg. 9: 925-31.

[9]. Brunelli A, Charloux A, Bolliger CT, Rocco G, Sculier J-P, et al., (2009) European Respiratory Society and European Society of Thoracic Surgeons joint task force on fitness for radical therapy. ERS/ESTS clinical guidelines, on fitness for radical in lung cancer patients (surgery and chemo-radiotherapy). Eur Respir J. 34(1): 17-41.

[10]. Quanger PH, Tammeling GI, Cotes JE, Pedersen OF , Peslin R , et al., (1993) Lung volumes and forced flows. Report working party Standardization of lung function tests. European Community for Steel and Coal. Official statement of the European Respiratory Society: Euro Respir J. 16 (6): $5-40$.
[11]. Gimenez Manuel (1992) The SWEET test for traning and measurement of endurance: I- physiological and biochemical effects; II .- application and results in normal and handicapped man. Thesis Nancy University : France. 1060.

[12]. Borg GA (1982) Psychophysical bases of perceived exert. Med Sci Sports Exerc. 14(5): 377-81.

[13]. Gimenez M, Saavedra P, Lantarón EM, Tran J, Martin N, et al., (2017) Comparison of Two Stairs Climbing Protocols for Smokers. Int J Resp Dis Care Med. 2(1): 8-13.

[14]. Laird NM, Ware JH (1982) Random-effects models for longitudinal data. Biometrics. 38(4): 963-74.

[15]. R Core Team (2013) R: A language and environment for statistical computing. R. Foundation for Statistical Computing, Vienna, Austria.

[16]. Pollock M, Roa J, Benditt J, Celli B (1993) Estimation of ventilatory reserve by stair climbing: a study in patients with chronic airflow obstruction. Chest. 104(5): 1378-1383.

[17]. Koegelenberg CFN, Diacon AH, Irani S, Bolliger CT (2008) Stair climbing in the functional assessment of lung resection candidates. Respiration. 75(4): 374-379.

[18]. Swinburne CR, Wakefield JM, Jones PW (1985) Performance, ventilation, and oxygen consumption in three different types of exercise tests in patients with chronic obstructive lung disease. Thorax. 40(8): 581-586.

[19]. Brunelli A, Al Refai M, Monteverde M, Borri A, Salati M, et al., (2002) Stair climbing test predicts cardiopulmonary complications after lung resection. Chest. 121(4): 1106-1110.

[20]. Gimenez M, Servera E, Saunier C, Lacoste J (1982) Square-Wave Endurance Exercise Test (SWEET) for training and assessment in trained and untrained subjects. II: Blood gases and acid-base balance. Eur J Appl Physiol. 49(3): 369-77.

[21]. Gimenez M, Cereceda V, Teculescu D, Aug F, Laxenaire MC (1982) SquareWave Endurance Exercise Test (SWEET) for training and assessment in trained and untrained subjects. III: Effect on $\mathrm{VO}_{2}$ max and maximal ventilation. Eur J Appl Physiol Occup Physiol. 49(3): 379-87.

[22]. Gimenez M, Abril E, Rodríguez F, Buono M (2004) Effects of SWEET raining on trained and untrained healthy subjects and on chronic lung patients. Prevention and Rehabilitation in chronic respiratory pathology: Physiotherapy, training and respiratory care. (2nd Edn), Madrid, Spain, Panamericana. 47-59, 103-117, 383-395.

[23]. Lonsdorfer J, Lampert E, Mettauer B, Hoppeler H, Frey M, et al., (2004) Physical after cardiac transplantation: A proposal for an endurance training program and assessment. Sci Sports. 7(1): 39-44.

[24]. Mettauer B, Lampert E, Schnedecker B, Bogui P, Lonsdorfer J (1993) A short endurance training program increases the physical fitness of heart transplant recipients. Sci Sports. 8: 25-6.

[25]. Lampert E, Mettauer B, Lonsdorfer J (1994) Effets d'un entrainement «en créneaux « sur le handicap physique tardif des greffés cardiaques. Méd Sport (Paris). 68: 142-44.

[26]. Chabot F, Siat J, Gimenez M, Polu JM (2004) Trasplante pulmonar: Ventilatory education and pre and post-operative muscle training. Prevention and Rehabilitation in chronic respiratory pathology: Physiotherapy, training and respiratory care. (2nd Edn), Madrid, Spain, Panamericana. 481-91.

[27]. Casanova C, Celli BR, Barria P, Casas A, Cote C, et al., (2011) The 6-min walk distance in healthy subjects: reference standards from seven countries. Eur Respir J. 37(1): 150-6.

[28]. Chetta A, Pisi G, Aiello M, Zani P, Olivieri D (2009) The walking capacity assessment in the respiratory patient. Respiration. 77(4): 361-7.

[29]. Rimmer JH, Rauworth AE, Wang EC, Nicola T, Hill B (2009) A preliminary study to examine the effects of aerobic and therapeutic (nonaerobic) exercise on cardiorespiratory fitness and coronary risk reduction in survivors. Arch Phys Med Rehab. 90(3): 407-12.

[30]. Nightingale EJ, Pourkazemi F, Hiller CE (2014) Systematic review of timed stair tests. J Rehabil Res Dev. 51(3): 335-350.

[31]. Hildebrand MW, Host HH, Binder EF, Carpenter B, Freedland KE, et al., (2012) Measuring treatment fidelity in a rehabilitation intervention study. Am J Phys Med Rehabil. 91(8): 715-724. 